\title{
AN EIGHT-MONTH MONITORING CAMPAIGN \\ ON A SAMPLE OF AGN
}

\author{
CLÁUDIA WINGE*,** and BRADLEY M. PETERSON \\ Dept. of Astronomy, The Ohio State University, USA \\ M.G. PASTORIZA** and T. STORCHI-BERGMANN** \\ Depto. de Astronomia, UFRGS, Brasil \\ and \\ J. BALDWIN \\ CTIO, Chile
}

We present the preliminary results of an 8-month monitoring campaign carried out on 6 AGN during the period December 1991 - July 1992. All but one of our targets showed continuum and/or line variability. The data were obtained using the 2D-Frutti + Cassegrain spectrograph at the CTIO 1.0-m telescope, and reduced following standard procedures. The slit width was $5^{\prime \prime}$ and the nuclear spectra were extracted in a $10^{\prime \prime}$ aperture. The wavelength coverage is $3500-7200 \AA$, with $8 \AA$ resolution. The data were flux calibrated using standard stars and then normalized using the [O III] $\lambda 5007 \AA$ line flux for each object. NGC 6814: our spectra reveal that this object is still in a low state of activity and within the $S / N$ ratio of our data, no variability was observed during this campaign. The stellar population is dominant in the nuclear spectrum and a synthesis using the star cluster library of Bica (1988) indicates a mainly old ( $\sim 86 \%$ of the continuum flux at $5870 \AA$ due to a population with age $\geq 10 \mathrm{Gyr}),\left[\mathrm{Z} / \mathrm{Z}_{\odot}\right] \geq 0.3$ stellar content, with an intrinsic reddening of $\mathrm{E}(\mathrm{B}-\mathrm{V})=0.20$. NGC 3227: using an off-nuclear spectrum corresponding to the two $5^{\prime \prime} \times 10^{\prime \prime}$ regions $20.4^{\prime \prime} \mathrm{E} / \mathrm{W}$ of the nucleus, we obtained also a mainly old $(77 \%$ at $5870 \AA$ with age $\geq 10 \mathrm{Gyr}),\left[\mathrm{Z} / \mathrm{Z}_{\odot}\right]=0.3$ ) synthetic stellar population, which contributes $\sim 43 \%$ of the nuclear light at $5600 \AA$. The cross correlation of the $4245 \AA$ continuum and $\mathrm{H} \beta$ light curves results in a 18 \pm 3 -day lag. IC 4329A: our data show evidence of variability as a slow and constant increase in both continuum and lines fluxes, but no isolated event was detected. ESO141-G55: the light curves show small variations in the continuum, but no noticeable line variability. Akn 120 and Fairall 9: the data consists of two sets of spectra, separated by $\sim 6$ months. Within each set little or no variability was detected, but strong line and continuum variations occurred between them.

* On leave from UFRGS, Brasil

** Visiting Astronomer at the Cerro Tololo Interamerican Observatory of the National Optical Observatories, operated by AURA under contract with the National Science Foundation

415

T. J.-L. Courvoisier and A. Blecha: Multi-Wavelength Continuum Emission of AGN, 415.

(C) 1994 IAU. Printed in the Netherlands. 